Short communication

\title{
The genetic legacy of Lonesome George survives: Giant tortoises with Pinta Island ancestry identified in Galápagos
}

\author{
Danielle L. Edwards ${ }^{\mathrm{a}, *}$, Edgar Benavides ${ }^{a}$, Ryan C. Garrick ${ }^{\mathrm{a}, \mathrm{b}}$, James P. Gibbs ${ }^{\mathrm{c}}$, Michael A. Russello ${ }^{\mathrm{d}}$, \\ Kirstin B. Dion ${ }^{a}$, Chaz Hyseni ${ }^{a}$, Joseph P. Flanagan ${ }^{e}$, Washington Tapia ${ }^{f}$, Adalgisa Caccone ${ }^{a}$ \\ a Department of Ecology and Evolutionary Biology, Yale University, New Haven, CT 06520, USA \\ ${ }^{\mathrm{b}}$ Department of Biology, University of Mississippi, MS 38677, USA \\ ${ }^{c}$ College of Environmental Science and Forestry, State University of New York, Syracuse, NY 13210, USA \\ ${ }^{\mathrm{d}}$ Department of Biology, University of British Columbia, Okanagan Campus, Kelowna, BC, Canada V1V 1V7 \\ ${ }^{\mathrm{e}}$ Houston Zoo, Houston, TX 77030, USA \\ ${ }^{\mathrm{f}}$ Galápagos National Park Service, Puerto Ayora, Galápagos, Ecuador
}

\section{A R T I C L E I N F O}

Article history:

Received 22 August 2012

Received in revised form 9 October 2012

Accepted 14 October 2012

\section{Keywords:}

Ancient DNA

Chelonoidis abingdoni

Conservation genetics

Galápagos giant tortoise

Hybridization

Lonesome George

\begin{abstract}
A B S T R A C T
The death of Lonesome George, the last known purebred individual of Chelonoidis abingdoni native to Pinta Island, marked the extinction of one of 10 surviving giant tortoise species from the Galápagos Archipelago. Using a DNA reference dataset including historical C. abingdoni and $>1600$ living Volcano Wolf tortoise samples, a site on Isabela Island known to harbor hybrid tortoises, we discovered 17 individuals with ancestry in C. abingdoni. These animals belong to various hybrid categories, including possible first generation hybrids, and represent multiple, unrelated individuals. Their ages and relative abundance suggest that additional hybrids and conceivably purebred C. abingdoni individuals still occur on Volcano Wolf. Spatial analyses suggest locations where additional individuals with $C$. abingdoni ancestry are most likely to be recovered, consistent with historical records of human movement of tortoises. These results provide an opportunity for species recovery of Pinta Island tortoises using individuals with $C$. abingdoni ancestry.
\end{abstract}

(c) 2012 Elsevier Ltd. All rights reserved.

\section{Introduction}

Prioritizing species for conservation is a process that integrates social, economic and cultural values with phylogenetic, biogeographic, ecological and demographic considerations (Arponen, 2012). Galápagos giant tortoises provide an example as they represent an evolutionary radiation with a diversity of morphotypes (Fritts, 1984), are top "mega-herbivores" that shape Galápagos ecosystems (Gibbs et al., 2010), and provide a charismatic draw for local tourism (Kenchington, 1989). This alignment of biological variation, ecological importance, and public interest propel giant tortoises as a major conservation priority in Galápagos (Cayot, 2008).

Perhaps the best-known Galápagos giant tortoise was Lonesome George, whose death on June 24th 2012 marked the extinction of Chelonoidis abingdoni, endemic to Pinta Island. His story exemplifies the plight of many other Galápagos tortoise species, as historical killing by whalers and buccaneers decimated popula-

* Corresponding author. Address: Department of Ecology and Evolutionary Biology, Yale University, 21 Sachem Street, New Haven, CT 06520, USA. Tel.: +1 7342392988.

E-mail address: danielle.edwards@yale.edu (D.L. Edwards). tions across Galápagos (Townsend, 1925; Pritchard, 1996). Four of the 14 named and taxonomically supported species (Poulakakis et al., 2012) went extinct over the last 200 years, with most remaining species considered endangered due to ongoing killing and introduced pests (Fig. 1a; Pritchard, 1996; Phillips et al., 2012).

As top "mega-herbivores" (Gibbs et al., 2008), seed dispersers (Blake et al., in press), and ecological engineers (Gibbs et al., 2010), giant tortoises provide ecosystem services that are critical to Galápagos ecosystems. As such, the complementarity of tortoise conservation and ecosystem recovery (Hamann, 1993) has helped align conservation goals for Galápagos (Jordán, 2009; Stouffer et al., 2012). Conservation management efforts seeking to recover and repatriate Galápagos giant tortoises have met with success using captive breeding programs (Milinkovitch et al., 2004, 2007, in press; Gibbs et al., 2008). However, the islands of Pinta, Floreana and Santa Fé remain without the tortoises that once inhabited them, threatening their ecological balance (Hamann, 1993).

Previous genetic studies reported that Volcano Wolf (VW, northern Isabela Island - Fig. 1a) hosts hybrid individuals with ancestry in two extinct tortoise species, C. abingdoni and C. elephantopus, the species once endemic to Floreana Island (Fig. 1a; Russello et al., 2007; Poulakakis et al., 2008). Since original reports were based on $<100 \mathrm{VW}$ samples, a subsequent expedition in 
(A)

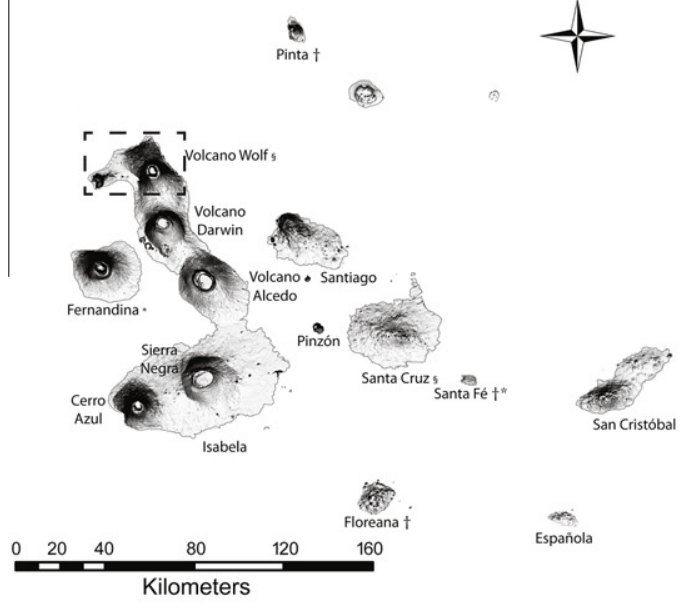

(B)

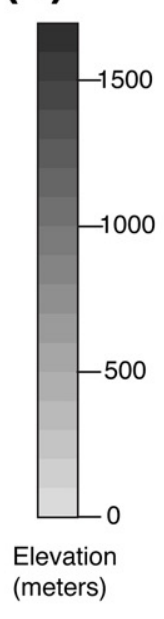

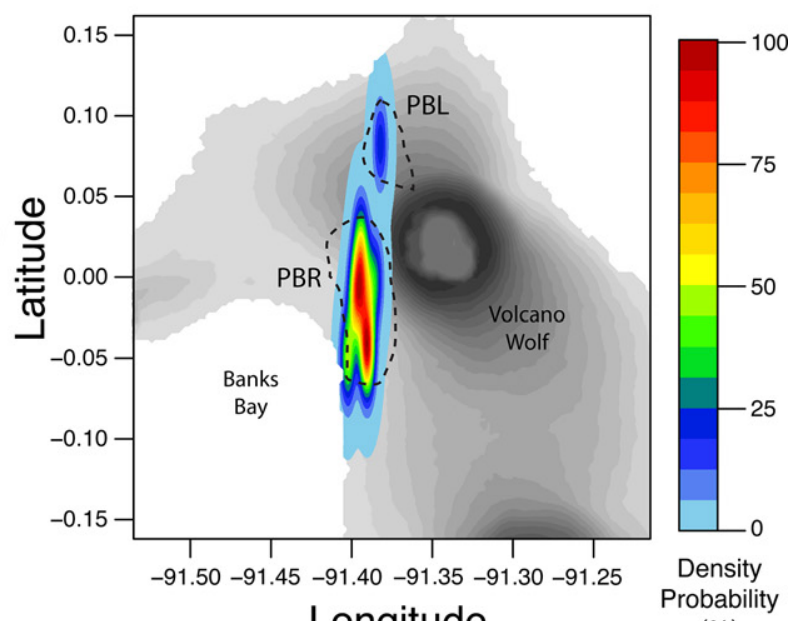

(\%)

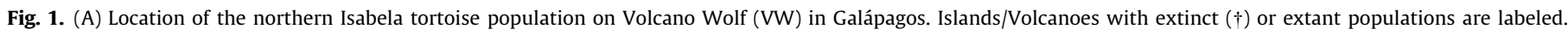

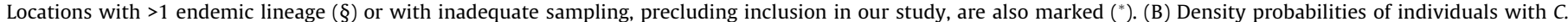
abingdoni ancestry showing the Puerto Bravo (PBR) and Piedras Blancas (PBL) VW tortoise distributions (dotted contours).

2008 intensively sampled this population (1667 samples), leading to the discovery of individuals with $C$. elephantopus ancestry (Garrick et al., 2012). Here we investigate the presence of $C$. abingdoni ancestry. Using the 2008 samples augmented by museum specimens and sub-fossil remains from Pinta Island, we report the detection of 17 individuals with C. abingdoni ancestry on VW.

\section{Methods}

\subsection{Samples and genetic methods}

Genetic data were separated into two datasets: a 'reference dataset' with 305 purebred individuals from 14 Galápagos Chelonoidis lineages (sensu Van Denburgh, 1914), and a 'test dataset' with 1667 tortoises of unknown ancestry sampled from VW in 2008 (Garrick et al., 2012). Blood, photos, and GPS coordinates were collected for each individual, which were assayed for variation at 12 dinucleotide microsatellite loci and a $\sim 700 \mathrm{bp}$ fragment of the mitochondrial (mtDNA) control region (Poulakakis et al., 2008; Benavides et al., 2012). Ancient DNA techniques were used to increase the C. abingdoni reference dataset from five (Russello et al., 2007) to twenty individuals (Table S1) using sub-fossil remains and museum specimens from Pinta Island (Supplementary Material).

\subsection{Detecting C. abingdoni ancestry on VW}

To establish the number of genetic clusters within the reference dataset, genotypic clustering analyses were run for $K=9-17$, covering a range of $K$-values identified in previous studies (Russello et al., 2007, 2010; Poulakakis et al., 2008; Garrick et al., 2012). STRUCTURE v2.3.3 (Pritchard et al., 2000) was run for $1 \times 10^{6}$ generations with a burn-in of $1 \times 10^{5}$ and five replicate runs using a location prior. Results were processed using structureHarvester.py (Earl and vonHoldt, 2012), and replicate runs summarized using CLUMPP (Jakobsson and Rosenberg, 2007). The $\Delta K$ metric (Evanno et al., 2005) identified an optimal value of $K=12$, as previously reported (Garrick et al., 2012). Genetic assignments ( $Q$-values) of the $1667 \mathrm{VW}$ test individuals were then estimated in relation to the 12 reference genetic clusters. Threshold $Q$-values $\left(Q_{T}\right)$ for ancestry established in both simulation (Vaha and Primmer, 2006) and empirical studies (Lancaster et al., 2006) were used to conservatively establish non-negligible levels of ancestry in C. abingdoni
$\left(Q_{T}>0.1\right)$. Levels of relatedness among $C$. abingdoni hybrids were assessed using ML-Relate (Kalinowski et al., 2006).

\subsection{Characterizing C. abingdoni ancestry on VW}

Simulations were used to establish the genetic makeup for each parent of VW hybrid individuals with ancestry in $C$. abingdoni. Theoretical expectations (e.g., $F_{1}$ hybrids should have $Q$-values $\sim 0.50$ in each of two parental clusters) were used to identify the crosses generating hybrids with C. abingdoni ancestry. Genotypes for 500 individuals were simulated in each hybrid classes using HYBRIDLAB (Nielsen et al., 2006) and analyzed in STRUCTURE to characterize $Q$-value frequency distributions. The approach of Garrick et al. (2012) was used to define criteria for hybrid classification. Theoretical predictions suggested five parental crosses were involved in generating individuals with $C$. abingdoni ancestry (Table S2). We implemented strict criteria for pedigree classification requiring all $Q$-value ranges and $Q_{D}$ (difference between $Q$-values for each parental genome contributing to cross) values to fall within the simulations specified range. Alternatively, we applied a majorityrule approach that allowed most but not all criteria to be met. In this latter case, a cross was considered probable if no more than two $Q$-value ranges and/or $Q_{D}$ values that fell outside of the simulated distribution.

\subsection{Age of individuals with C. abingdoni ancestry}

Age estimation of VW individuals with $C$. abingdoni ancestry, combined with pedigree determination, allowed evaluation of the likelihood that purebred C. abingdoni individuals still live on VW. Scute (plate on carapace) growth ring presence and scute smoothness (from photographs - Figs. S1-S14) were used along with carapace length to estimate age (Table S3). For individuals with obvious scute rings, ring counts could be used to estimate age. With advancing age, tortoise scutes are worn smooth, suggesting individuals are older adults. Where no photo was available, we used carapace length to assign these individuals to broad age categories, as size and age are correlated. Given the difficulties in rigorously defining age-related categories using scute counts (Wilson et al., 2003), we conservatively categorized tortoises only into three biologically meaningful categories: "Juvenile"(<20 years), "Adult" (2055 years), and "Old Adult" (>55 years; Tables S5 and S6). 


\subsection{Spatial clustering of C. abingdoni ancestry on VW}

Two-dimensional density kernel estimation (Venables and Ripley, 2002) was employed to identify areas on VW with the highest probability of containing individuals with $C$. abingdoni ancestry. Density probability estimates were calculated using GPS coordinates (Table S4) of individuals with $C$. abingdoni ancestry using the ke2d function of the MASS package (Venables and Ripley, 2002) in the $R$ statistical software package (http://www.r-project.org/). These density estimates were then plotted on a digital elevation model for northern Isabela Island.

\section{Results}

We identified 17 hybrids with non-negligible levels of $C$. abingdoni ancestry $\left(Q>Q_{T}\right)$, including nine females, three males, and five juveniles. Some, but not all, individuals are related (Table S5), being either full or half siblings. No parent-offspring relationships were detected and none were inferred as close-relatives of Lonesome George. Under strict criteria for pedigree assignment, two hybrids could be unambiguously assigned to a specific parental cross, whereas an additional four individuals were considered probable using the majority-rule approach (Tables S6-7). Of these individuals, one was considered an $F_{1}$ hybrid with a purebred $C$. abingdoni parent under strict assignment while another three were identified in this hybrid category under majority-rule assignment. It should be noted here that STRUCTURE has statistical limitations in distinguishing between $F_{1}$ and $F_{2}$ hybrids in this system (see Russello et al., 2007). Thus, given that potential $F_{1}$ individuals contain three or more parental gene pools (Table $\mathrm{S} 6$ ), these individuals could either be $F_{1}$ hybrids or $F_{2}$ hybrids between two Pinta $F_{1}$ hybrids with different ancestries. Regardless, these individuals contain a significant proportion of Pinta ancestry. The parental crosses involved in generating the other $11 \mathrm{C}$. abingdoni hybrids were ambiguous. The detected hybrid individuals contain ancestries in eight additional species, including those from Pinzón, Northern Isabela (Volcano Wolf both PBL and PBR), Santa Cruz, Española, Santiago, Southern Isabela and Floreana Islands (Fig. 1a). The estimated age of the 17 hybrids ranged from juvenile to old adult, with four juveniles, five adults, five old adults and three individuals of uncertain age.

We found four mtDNA haplotypes in the 17 C. abingdoni hybrids (Table S7), none shared Lonesome George's haplotype, while 75\% had a C. hoodensis-like haplotype (Russello et al., 2007). This haplotype is close (three substitutions away) to the single haplotype represented in living $C$. hoodensis tortoises from Española Island and quite distant from the closest $C$. abingdoni haplotype ( $\geqslant 16$ substitutions away: Poulakakis et al., 2008). Two of the remaining three haplotypes had previously been detected in native VW tortoises ( $C$. becki) and its sister taxon from Santiago island (C. darwini), possibly due to hybridization between $C$. becki and $C$. abingdoni tortoises. The fourth haplotype was previously found in $C$. elephantopus (Floreana Island), likely the result of hybridization on VW between C. abingdoni and C. elephantopus individuals.

Spatial analysis revealed a concentration of $C$. abingdoni (Fig. 1b) hybrids near Banks Bay around the VW Puerto Bravo (PBR) population of tortoises; however, hybrid individuals were also found on the northern slopes of VW near Piedras Blancas (PBL).

\section{Discussion}

Here we report the discovery of 17 individuals living on VW that have genetic ancestry in Lonesome George's species, C. abingdoni. The majority of individuals have mtDNA more closely related to $C$. hoodensis than to other $C$. abingdoni mtDNA, consistent with previous studies (Russello et al., 2007). Historically, this pattern suggests that this mtDNA haplotype may have once been shared by both species, as $C$. abingdoni and $C$. hoodensis are sister taxa (Caccone et al., 2002; Poulakakis et al., 2008). On a finer-scale, these findings indicate that the majority of hybrids on $\mathrm{VW}$ acquired $C$. abingdoni ancestry from their mothers.

Although we identified no parent-offspring relationships among the 17 hybrid individuals, some individuals were inferred to be full or half-siblings and many were inferred to have different ancestries to eight other species with up to four parental species involved in any one cross (Table S7). This precludes the use of more sensitive methods of hybrid assignment that are limited to the inclusion of only two parental genomes (e.g., Anderson and Thompson, 2002). Hybrid assignment is also difficult with only 12 loci (Vaha and Primmer, 2006). Due to these limitations, we were able to identify the genetic crosses accounting for the ancestry of only six individuals (Table S7). However, the genetic contribution of a $C$. abingdoni animal to the 17 hybrids was clear regardless of the hybrid category, as we found four tortoises may have had a purebred $C$. abingdoni as a parent, and 13 tortoises are likely to have one $F_{1} C$. abingdoni parent. These individuals may be valuable to future captive breeding strategies. Moreover, the ongoing development of SNP markers for these species should improve diagnostic abilities.

The ages of $C$. abingdoni hybrids range from juvenile to old adult (Table S7). Since these species live for $>100$ years and reproduce throughout their lives (Paré and Lentini, 2010), our results suggest that purebred parents may still be alive and in breeding condition on VW. Interestingly, a $C$. abingdoni $F_{1}$ parent appears to be involved in the cross that produced hybrid individuals in all age classes, including juveniles. Such individuals with high levels of Pinta ancestry can provide avenues for reestablishing the $C$. abingdoni lineage. Moreover, given current VW sampling represents $\sim 20 \%$ of the population (Garrick et al., 2012) and a detection rate of $\sim 1.02 \%$ (17/1667), it is likely that an additional $\sim 60-70$ C. abingdoni hybrids are yet to be discovered on VW.

The geographic clustering of $C$. abingdoni hybrids in Banks Bay has implications for the origin of $C$. abingdoni alleles on VW. With prevailing northwesterly ocean currents (Chelton et al., 2004) and assuming a passive overwater dispersal scenario (Parent et al., 2008), spatial distribution of $C$. abingdoni ancestry should be concentrated on the eastern coast of VW near PBL rather PBR (Fig. 1b). Interestingly, PBR is proximate to Banks Bay, a natural harbor for naval and whaling vessels (Porter, 1822; Townsend, 1925), where tortoises were thrown overboard when no longer needed (Porter, 1822). This historical scenario is also consistent with previous results showing the genetic contribution of multiple non-native species to the VW tortoises (Russello et al. 2007; Garrick et al. 2012).

Whereas hybridization and human-mediated dispersal are regarded as threats to biodiversity (Allendorf et al., 2001), we highlight potential unforeseen conservation benefits from such events for Galápagos giant tortoises. Given the ecological importance of these species (Hamann, 1993; Gibbs et al., 2008), the use of hybrids in captive breeding programs may be the only option to re-introduce a key ecosystem engineer while preserving the evolutionary legacy of extinct endemics. Here, we have shown that individuals carrying $C$. abingdoni alleles are still alive on VW and speculate that as-yet undetected individuals, possibly purebreds, can be found around Banks Bay (Fig. 1b).

The next step will be to further sample the VW tortoise population to recover these individuals of conservation importance and possibly find new ones. Hybrid removal would also benefit the genetic integrity of the native VW lineages while enabling the inclusion of greater numbers of hybrid individuals in recovery efforts, thus mitigating inbreeding impacts. Moreover, the identification 
of additional individuals with higher levels of Pinta ancestry may allow for tiered management programs permitting immediate release for ecological restoration of "lower" quality hybrids onto Pinta, and retaining "higher" quality hybrids for captive breeding followed by offspring release, as for the $C$. hoodensis recovery program (Milinkovitch et al., 2004, 2007, in press). This strategy could enhance the retention of endemic species' genes in the restored population, while kick-starting the ecological recovery of the island. Its success, however, will be contingent on the reproductive viability, diversity, and familial relationships of the recovered hybrids.

Driven by our findings for $C$. abingdoni and the previous detection of C. elephantopus (Poulakakis et al., 2008; Garrick et al. 2012) ancestry on VW, planning is now underway for an expedition to recover these individuals of conservation importance and identify new ones. The outcomes from the spatial analysis provide a focus for these efforts: the western slopes of VW (Fig. 1b). Our discovery lends considerable hope and practical guidance for conservation efforts aiming to reestablish a tortoise population on Pinta Island with close genetic affinity to $C$. abingdoni despite the recent death of Lonesome George.

\section{Acknowledgements}

This work is dedicated to Lonesome George. The Galápagos National Park Service and Charles Darwin Research Station were instrumental in supporting logistics. We thank B. Kajdacsi, L. Márquez and S. Bahan for help with data collection. Financial support came from the Charles Darwin Foundation, Galápagos National Park Service, Bay and Paul Foundation, Eppley Foundation, Galápagos Conservancy, National Geographic Society, Turtle Conservation Fund, and Yale Institute for Biospheric Studies. Permits: CITES permit - 12US209142/9, and collection permit (PC-02-12) from the Galápagos National Park Service.

\section{Appendix A. Supplementary material}

Supplementary data associated with this article can be found, in the online version, at http://dx.doi.org/10.1016/j.biocon.2012.10.014. These data include Google maps of the most important areas described in this article.

\section{References}

Allendorf, F.W., Leary, R.F., Spruell, P., Wenburg, J.K., 2001. The problems with hybrids: setting conservation guidelines. Trends Ecol. Evol. 16, 613-622.

Anderson, E.C., Thompson, E.A., 2002. A model-based method for identifying species hybrids using multi-locus genetic data. Genetics 160, 1217-1229.

Arponen, A., 2012. Prioritizing species for conservation planning. Biodivers. Conserv. 21, 393-875.

Benavides, E., Russello, M.A., Boyer, D., Wiese, R.J., Kajdacsi, B., Márquez, L., Garrick, R.C., Caccone, A., 2012. Lineage identification and genealogical relationships among captive Galápagos tortoises. Zoo. Biol. 31, 101-120.

Blake, S., Wikelski, M., Cabrera, F., Guezou, A., Silva, M., Sadeghayobi, E., Yackulic, C.B., Jaramillo, P., in press. Seed dispersal by Galápagos tortoises. J. Biogeog. (doi:10.1111/j.1365-2699.2011.02672.x).

Caccone, A., Gentile, G., Gibbs, J.P., Fritts, T.H., Snell, H.L., Betts, J., Powell, J.R., 2002. Phylogeography and history of giant Galápagos tortoises. Evolution 56, 20522066.

Cayot, L.J., 2008. The restoration of giant tortoises and land iguana populations in Galápagos. Galápagos Res. 65, 39-43.

Chelton, D.B., Schlax, M.G., Freilich, M.H., Milliff, R.F., 2004. Satellite measurements reveal persistent small-scale features in ocean winds. Science 303, 978-983.

Earl, D.A., vonHoldt, B.M., 2012. STRUCTURE HARVESTER: a website and program for visualizing STRUCTURE output and implementing the Evanno method. Conserv. Genet. Resour. 4, 359-361.

Evanno, G., Regnaut, S., Goudet, J., 2005. Detecting the number of clusters of individuals using the software STRUCTURE: a simulation study. Mol. Ecol. 14 2611-2620.
Fritts, T.H., 1984. Evolutionary divergence of giant tortoises in Galápagos. Biol. J. Linn. Soc. 21, 165-176.

Garrick, R.C., Benavides, E., Russello, M.A., Gibbs, J.P., Poulakakis, N., Dion, K.B. Hyseni, C., Kajdacsi, B., Márquez, L., Bahan, S., Ciofi, C., Tapia, W., Caccone, A. 2012. Genetic rediscovery of an 'extinct' Galápagos giant tortoise species. Curr. Biol. 22, R10-R11.

Gibbs, J.P., Marquez, C., Sterling, E.J., 2008. The role of endangered species reintroduction in ecosystem restoration: tortoise-cactus interactions on Española Island. Galápagos Rest. Ecol. 16, 88-93.

Gibbs, J.P., Sterling, E.J., Zabala, F.J., 2010. Giant tortoises as ecological engineers: a long-term quasi-experiment in the Galápagos Islands. Biotropica 42, 208-214.

Hamann, O., 1993. On vegetation recovery, goats and giant tortoises on Pinta Island, Galápagos, Ecuador. Biodivers. Conserv. 2, 138-151.

Jakobsson, M., Rosenberg, N.A., 2007. CLUMPP: a cluster matching and permutation program for dealing with label switching and multimodality in analysis of population structure. Bioinformatics 23, 1801-1806.

Jordán, F., 2009. Keystone species and food webs. Phil. Trans. R. Soc. B 364, $1733-$ 1741.

Kalinowski, S.T., Wagner, A.P., Taper, M.L., 2006. ML-RELATE: a computer program for maximum likelihood estimation of relatedness and relationship. Mol. Ecol. Notes 6, 576-579.

Kenchington, R.A., 1989. Tourism in the Galápagos Islands: the dilemma of conservation. Environ. Conserv. 16, 227-232.

Lancaster, M.L., Gemmell, N.J., Negro, S., Goldsworthy, S., Sunnucks, P., 2006 Ménage à trois on Macquarie island: hybridization among three species of fur seal (Arctocephalus spp.) following historical population extinction. Mol. Ecol. 15, 3681-3692.

Milinkovitch, M.C., Monteyne, D., Gibbs, J.P., Fritts, T.H., Tapia, W., Snell, H.L., Tiedmann, R., Caccone, A., Powell, J.R., 2004. Genetic analysis of a successful repatriation programme: giant Galápagos tortoises. Proc. Roy. Soc. Lond. 271, 341-345.

Milinkovitch, M.C., Monteyne, D., Russello, M., Gibbs, J.P., Snell, H.L., Tapia, W., Marquez, C., Caccone, A., Powell, J.R., 2007. Giant Galápagos tortoises: molecular genetic analysis reveals contamination in a repatriation program of an endangered taxon. BMC Ecol. 7, 2.

Milinkovitch, M.C., Kanitz, R., Tiedemann, R., Tapia, W., Llerena, F., Caccone, A. Gibbs, J.P., Powell J.R., in press. Recovery of a nearly extinct galápagos tortoise despite minimal genetic variation. Evol. Appl. http://dx.doi.org/10.1111/ eva.12014.

Nielsen, E.E., Bach, L.A., Kotlicki, P., 2006. HYBRIDLAB (Version 1.0): a program for generating simulated hybrids from population samples. Mol. Ecol. Notes 6, 971 973.

Paré, J.A., Lentini, A.M., 2010. Reptile geriatrics. Vet. Clin. Exot. Anim. 13, 15-25.

Parent, C.E., Caccone, A., Petren, K., 2008. Colonization and diversification of Galápagos terrestrial fauna: a phylogenetic and biogeographical synthesis. Phil. Trans. Roy. Soc. B: Biol. Sci. 363, 3347-3361.

Phillips, R.B., Widenfeld, D.A., Snell, H.L., 2012. Current status of alien vertebrates in the Galápagos Islands: invasion history, distribution, and potential impacts. Biol. Invas. 14, 461-480.

Porter, D., 1822. Journal of a cruise made to the Pacific Ocean. J \& J Harper, New York, USA.

Poulakakis, N., Glaberman, S., Russello, M.A., Beheregaray, L.B., Ciofi, C., Powell, J.R. Caccone, A., 2008. Historical DNA analysis reveals living descendants of an extinct species of Galápagos tortoise Proc. Natl. Acad. Sci. USA 105, 1546415469.

Poulakakis, N., Russello, M.A., Geist, D., Caccone, A., 2012. Unravelling the peculiarities of island life: vicariance, dispersal and the diversification of the extinct and extant giant Galápagos tortoises. Mol. Ecol. 21, 160-173.

Pritchard, J.K., Stephens, M., Donnelly, P., 2000. Inference of population structure using multi-locus data. Genetics 155, 945-959.

Pritchard, P.C.H., 1996. The Galápagos Tortoises: Nomenclatural and Survival Status, Chelonian Research Monographs no. 1. Chelonian Research Foundation, Lunenburg MA, USA.

Russello, M.A., Beheregaray, L.B., Gibbs, J.P., Fritts, T., Havill, N., Powell, J.R., Caccone, A., 2007. Lonesome George is not alone among Galápagos tortoises. Curr. Biol. 17, R317-R318.

Russello, M.A., Poulakakis, N., Gibbs, J.P., Tapia, W., Benavides, E., Powell, J.R., Caccone, A., 2010. DNA from the past informs ex situ conservation for the future: an 'extinct' species of Galápagos tortoise identified in captivity. PLoS ONE 5 e8683.

Stouffer, D.B., Sales-Pardo, M., Sirer, M.I., Bascompte, J., 2012. Evolutionary conservation of species' roles in food webs. Science 335, 1489-1492.

Townsend, C.H., 1925. The Galápagos Tortoises in Relation to the Whaling Industry. New York Zoological Society, New York, USA.

Vaha, J.P., Primmer, C.R., 2006. Efficiency of model-based Bayesian methods for detecting hybrid individuals under different hybridization scenarios and with different numbers of loci. Mol. Ecol. 15, 63-72.

Van Denburgh, J., 1914. The Gigantic Land Tortoises of the Galápagos Archipelago. California Academy of Sciences, San Francisco, USA.

Venables, W.N., Ripley, B.D., 2002. Modern Applied Statistics with S, fourth ed Springer, New York, USA.

Wilson, D.S., Tracy, C.R., Tracy, C.R., 2003. Estimating age of turtles from growth rings: a critical evaluation of the technique. Herpetologica 59, 178-194. 